\title{
How do I rule out aortic dissection?
}

\author{
Robert Ohle, MBBCh, MA, MSc ${ }^{1,3}$; Sarah Mclsaac, MBBCh, MEd, BSc ${ }^{2,3}$; \\ Paul Atkinson, (Ed.), MB MA
}

\section{CLINICAL SCENARIO}

A 75 -year-old male presents with gradual onset central chest pain radiating to his back. Past medical history is significant for hypertension. Does he need a computed tomography (CT) scan to rule out acute aortic dissection?

\section{KEY CLINICAL QUESTIONS}

\section{How often is acute aortic dissection misdiagnosed? \\ 1. Acute aortic dissection presents with a myriad of symptoms that overlap with more common diagnoses, and this can lead to a misdiagnosis in $14 \%-38 \%$ of cases.}

Misdiagnosis has decreased from a staggering $98 \%$ diagnosed at postmortem in 1934, to $14 \%-38 \%$ in recent years. ${ }^{1}$ The most common initial misdiagnoses are acute coronary syndrome, pulmonary embolism, and stroke. Patients with these suspected diagnoses should also be screened for highrisk features of acute aortic dissection. If none are present, they are unlikely to have an acute aortic dissection. If high-risk features are present, balance your clinical suspicion for an aortic dissection with the likelihood of an alternative diagnosis using an approach such as RAPID² (see Box 1).

\section{Box 1. RAPID assessment of high-risk features for an acute aortic dissection}

o Risk factors: connective tissue disease, aortic aneurysm, family history of aortic disease, bicuspid aortic valve

o Alternative diagnosis: (that mimics acute aortic dissection) acute coronary syndrome, pulmonary embolism, or stroke

o Physical exam: hypotension, pulse deficit, new murmur, or neurological deficit

o Impression: clinical suspicion for acute aortic dissection

o Description of pain: migrating, tearing, sharp, thunderclap, severe, worst ever

Think about acute aortic dissection if any are present.

\section{Can clinical assessment alone rule out acute aortic dissection?}

2. A likely alternative diagnosis, or a low clinical suspicion, rules out acute aortic dissection $99 \%$ of the time.

A large prospective trial found that, in patients with an initial clinical suspicion of acute aortic dissection, clinical judgement without a CT scan reduced the probability of acute aortic dissection to $0.7 \%$. A low volume centre will see 5-8 cases per year, and a high volume referral centre will see $20 /$ year. Although the percentage of cases misdiagnosed is high, the absolute number of aortic dissections is very low. Clinical reasoning alone has the potential to take you below your test threshold.

From the ${ }^{1}$ Department of Emergency Medicine, Northern Ontario School of Medicine, Sudbury, ON; ${ }^{2}$ Division of Critical Care, Department of Medicine, Northern Ontario School of Medicine, Sudbury, ON; ${ }^{3}$ Health Science North Research Institute, Sudbury, ON; and ${ }^{4}$ Dalhousie University, Saint John Regional Hospital, Saint John, NB.

*Correspondence to: Dr. Robert Ohle, Health Science North Research Institute, Health Science North, 41 Ramsey Lake Rd., Sudbury, ON P3E 5J1; Email: Robert.ohle@gmail.com

(C) Canadian Association of Emergency Physicians

CJEM 2019;21(1):34-36

DOI 10.1017/cem.2018.484 


\section{Does the absence of high-risk features rule out the diagnosis of acute aortic dissection?}

The absence of any high-risk feature for acute aortic dissection makes the diagnosis unlikely.

The American Heart Association (AHA) developed an evidence informed expert consensus clinical decision aid in 2010 (Table 1). Nazerian et al. ${ }^{3}$ recently published the results of a prospective study looking at the combination of this rule and D-dimer testing. Using the rule alone would have missed $5 \%$ of cases. ${ }^{3}$ Rogers et al. ${ }^{4}$ retrospectively applied it to the 2,500-case International Registry of Acute Aortic Dissection (IRAD) database in 2011. They found that $4 \%$ of the cases would be missed using the rule alone, reduced to $2.7 \%$ in combination with a negative chest X-ray. ${ }^{4}$ The six academic hospitals in Nazerian et al.'s study averaged 20 cases each per year, so $4 \%$ translates to 1 missed case every year. ${ }^{3}$ For non-referral centres, $4 \%$ translates to 1 every 3 years. So out of the tens of thousands of patients who are seen over a 3 -year period, only 1 would be a missed acute aortic dissection. ${ }^{3}$

\begin{tabular}{|c|c|c|}
\hline History & Risk factor & Physical exam \\
\hline $\begin{array}{l}\text { - Acute onset pain } \\
\text { - Severe pain } \\
\text { - Tearing/ripping pain }\end{array}$ & $\begin{array}{l}\text { - Connective tissue disease } \\
\text { - Bicuspid aortic valve } \\
\text { - Aortic aneurysm } \\
\text { - Recent aortic manipulation }\end{array}$ & $\begin{array}{l}\text { - Hypotension } \\
\text { - Pulse deficit/blood pressure differential } \\
\text { - Murmur of aortic insufficiency } \\
\text { - Neurological deficit }\end{array}$ \\
\hline
\end{tabular}

\section{Can D-dimer testing help rule out acute aortic dissection in this patient? \\ 4. In the absence of a proven diagnostic algorithm, it is difficult to know whether D-dimer will improve the miss rate or just increase the number of imaging studies performed.}

D-dimer has a sensitivity ranging from $90 \%-100 \%$ and a specificity of around $60 \%$. Similar to the use in testing for pulmonary embolism, if the clinical suspicion is high, a negative D-dimer does not rule out the diagnosis; if your suspicion is extremely low, a negative D-dimer will have little utility in lowering the probability of the condition any further. As of yet, we do not have any good criteria to define which patients might benefit from D-dimer testing to reduce risk below the threshold for CT. The European Society of Cardiology supports its use as part of the overall assessment. The AHA guidelines from 2010 do not support its use, and the American College of Emergency Physicians (ACEP) states it should not be used in isolation. When studied in patients with an ADD-RS $\leq 1$, this strategy proved to be quite sensitive, only missing $1.2 \%$ of all cases. However, implementation of this pathway could potentially lead to an increase in imaging without any proven benefit in reducing the miss rate. ${ }^{5-7}$

\section{If an acute aortic dissection is suspected, what is the imaging modality of choice? \\ 5. Acute aortic dissection can be accurately diagnosed by a CT scan with intravenous contrast of the chest and abdomen.}

CT is the initial imaging of choice to diagnose aortic dissection in the majority of patients. There is similar diagnostic accuracy for magnetic resonance imaging and transesophageal echocardiography with a sensitivity of $98 \%-100 \%$ and specificity of $95 \%-98 \% .^{5}$ Transthoracic echocardiography (TTE) cannot rule out acute aortic dissection but has the ability to rule it in if a dissection flap is seen. The sensitivity and specificity of TTE ranges from $77 \%-80 \%$ and $93 \%-96 \%$, respectively, for the involvement of the ascending aorta. However, TTE is successful in detecting a distal dissection of the thoracic aorta in only $70 \%$ of patients. ${ }^{5,7}$ 


\section{When should treatment be initiated for an acute aortic dissection? \\ 6. Initial management of a confirmed case involves expedited surgical referral and immediate symptom (pain/nausea), heart rate, and blood pressure control.}

Treatment is aimed at reducing propagation of the dissection along the aorta by controlling shear stress. If there is any suspicion, treatment should begin with aggressive symptom control of pain and nausea. If there is a high probability or confirmed diagnosis, then control of the heart rate $(<60 \mathrm{bpm})$ should be initiated. Beta-blockers are the initial medication of choice and have been associated with a decrease in mortality. ${ }^{5}$ If after heart rate control the systolic blood pressure is ?>? $120 \mathrm{~mm} \mathrm{Hg}$, a vasodilator should be initiated (target systolic blood pressure of 100-120 mm Hg).

\section{CASE RESOLUTION}

An electrocardiogram and troponins are negative. You get a chest X-ray that is also normal. An aortic dissection is possible, but the probability is so low that no further workup is required.

\section{KEY POINTS}

1. There is no current validated clinical decision rule that, in isolation, can rule out the presence of acute aortic dissection.

2. Clinical features can help decide which patients are low risk; however, definitive investigation by a CT is required if an acute aortic dissection is under serious consideration, and there is not an alternative diagnosis that could explain the patient's symptoms.

3. Acute aortic dissection is a rare presentation to the emergency department that should be considered in your differential, because the mortality rate is significant if not diagnosed in a timely manner.

Keywords: Aortic dissection, chest pain, emergency medicine

Competing interests: None declared.

\section{REFERENCES}

1. Hirst AE JR, Varner J, Kime SW JR. Dissecting aneurysm of the aorta: a review of 505 cases. Medicine. $1958 ; 37(3): 217$.

2. Hansen MS, Nogareda GJ, Hutchison SJ. Frequency of and inappropriate treatment of misdiagnosis of acute aortic dissection. Am 93 7 Cardiol. 2007;99(6):852-6.

3. Nazerian P, Mueller C, de Matos Soeiro A, et al. Diagnostic accuracy of the aortic dissection detection risk score plus D-dimer for acute aortic syndromes: the ADvISED Prospective Multicenter Study. Circulation 2017: CIRCULATIONAHA. 117.029457.

4. Rogers AM, Hermann LK, Booher AM, et al. Sensitivity of the aortic dissection detection risk score, a novel guideline-based tool for identification of acute aortic dissection at initial presentation: results from the International Registry of Acute Aortic Dissection. Circulation 2011;123(20):2213-8.

5. Hiratzka LF, Bakris GL, Beckman JA, et al. 2010 ACCF/AHA/AATS/ACR/ASA/SCA/SCAI/SIR/STS/SVM guidelines for the diagnosis and management of patients with thoracic aortic disease. 7 Am Coll Cardiol 2010;55(14):e27-e129.

6. Erbel R, Aboyans V, Boileau C, et al. 2014 ESC Guidelines on the diagnosis and treatment of aortic diseases. Eur Heart $72014 ; 35$ (41):2873-2926.

7. Diercks DB, Promes SB, Schuur JD, Shah K, Valente JH, Cantrill SV. Clinical policy: critical issues in the evaluation and management of adult patients with suspected acute nontraumatic thoracic aortic dissection. Ann Emerg Med 2015;65(1):32-42. e12.

8. Strauss C, Harris K, Hutchison S, et al. "TIME IS LIFE”: EARLY MORTALITY IN TYPE A ACUTE AORTIC DISSECTION: INSIGHTS FROM THE IRAD REGISTRY. 7 Am Coll Cardiol 2013;61(10 Suppl):E1516. 